\title{
Isolation of non-sporing anaerobic rods from infections in children
}

\author{
I. BROOK* \\ Department of Pediatrics, Georgetown University School of Medicine, Washington DC, USA
}

From 1974 to 1994, 2033 microbiological specimens from children were submitted for cultures for anaerobic bacteria. Fifty-seven isolates of Bifidobacterium spp. were obtained from $55(3 \%)$ children, 67 isolates of Eubacterium spp. from $65(3 \%)$ children and 41 isolates of Lactobacillus spp. from 40 (2\%) children. Most Bifidobacterium isolates were from chronic otitis media, abscesses, peritonitis, aspiration pneumonia and paronychia. Most Eubacterium isolates were from abscesses, peritonitis, decubitus ulcers and bites. Lactobacillus spp. were mainly isolated from abscesses, aspiration pneumonia, bacteraemia and conjunctivitis. Most $(>90 \%)$ infections from which these species were isolated were polymicrobial and yielded a mixture of aerobic and anaerobic bacteria. The organisms most commonly isolated with the non-sporing anaerobic gram-positive rods were Peptostreptococcus spp., Bacteroides spp., pigmented Prevotella and Porphyromonas spp., Fusobacterium spp., Staphylococcus aureus and Escherichia coli. Most Bacteroides spp. and $E$. coli were isolated from intra-abdominal infection and skin and soft tissue infection around the rectal area, whereas most Prevotella, Porphyromonas and Fusobacterium isolates were from oropharyngeal, pulmonary and head and neck sites. The predisposing conditions associated with the isolation of non-sporing anaerobic grampositive rods were previous surgery, malignancy, steroid therapy and immunodeficiency. Antimicrobial therapy was given to $149(83 \%)$ of the 160 patients, in conjunction with surgical drainage or correction of pathology in $89(56 \%)$.

\section{Introduction}

There has been increased interest in recent years about the role of anaerobic bacteria in paediatric infections [1]. The anaerobic species of most concern have been members of the genera Bacteroides, Clostridium, Fusobacterium and Peptostreptococcus [1]. Nonsporing, anaerobic, gram-positive rods such as Bifidobacterium, Eubacterium and Lactobacillus species are usually considered to be of relatively low virulence. These organisms are taxonomically unrelated but are all non-motile and catalase-negative. These organisms have been isolated from adults with pulmonary infection ( $B$. dentium) [2], from intra-uterine devices ( $E$. nodatum) [3], and from peritonitis and the bloodstream (Lactobacillus spp.) $[4,5]$. However, their pathogenic role in these and other infections is uncertain. The organisms have been isolated in pure culture in only a few instances and are usually isolated in mixed culture from clinical specimens $[1,6]$. The

Received 14 Sept. 1995; revised version accepted 2 Dec. 1995.

${ }^{*}$ Correspondence should be sent to Professor I. Brook, PO Box 70412, Chevy Chase, MD 20813-0412, USA. infections where they have been found most often are chronic otitus media and sinusitis, aspiration pneumonia, and intra-abdominal, obstetric and gynaecological and skin and soft-tissue infections $[1,6,7]$.

This retrospective review summarises my experience of the isolation of Bifidobacterium, Eubacterium and strictly anaerobic Lactobacillus spp. from infections in children over a 20 -year period. Some of the data have been published previously in articles describing the role of anaerobic bacteria in various paediatric infections [1], but cases not previously presented are also included, and the clinical associations of Bifidobacterium, Eubacterium and strictly anaerobic Lactobacillus spp. in infections in children are emphasised.

\section{Patients and methods}

\section{Patients}

The specimens included in this review were submitted for anaerobic investigation between June 1974 and June 1994 in the following hospitals: University of California Medical Center, County Medical Center and Serra 
Memorial Hospital in Los Angeles, and Fairview State Hospital, Costa Mesa, CA; Children's Hospital National Medical Center and South-East Community Medical Center in Washington DC; and the Naval Hospital in Bethesda, MD, USA. The clinical microbiology laboratory records were reviewed to identify patients from whom Bifidobacterium, Eubacterium and anaerobic Lactobacillus spp. were isolated and reported as potentially significant pathogens. Where available, the case records of the patients were reviewed to ascertain the presence and site of infection, associated microorganisms, underlying diseases and possible predisposing or associated conditions.

\section{Microbiological examination}

Only specimens that were collected properly without contamination by the normal skin or mucosal flora and submitted in transport media appropriate for anaerobic bacteria were accepted by the microbiology laboratories. These were generally specimens obtained during surgery or by aseptic needle or biopsy aspiration of abscesses or fluid from body cavities. Lung aspirates were obtained by transtracheal aspiration or through a tracheostomy or endotracheal tube or by biopsy. When possible, pus and fluids were collected and transported in syringes. Tissues were transported in oxygen-free gassed-out tubes. Swab specimens were submitted in the Port-A-Cul transport swab system (BBL, Cockeysville, MD, USA). However, precise records of all of the transport media used were not available. Blood for culture was collected aseptically from patients suspected of having bacteraemia and was inoculated $(10 \%)$ into the anaerobic bottle of one of two commercially produced blood culture broth media; both were under vacuum and with $\mathrm{CO}_{2} 5 \%$ in the atmosphere.

The specimens were inoculated on to pre-reduced vitamin $\mathrm{K}_{1}$-enriched Brucella Blood Agar (BBL), blood agar with kanamycin and vancomycin, blood agar containing colistin sulphate and nalidixic acid, and an enriched thioglycolate broth containing haemin and vitamin $\mathrm{K}_{1}[8,9]$. The cultures were incubated in GasPak jars (BBL) and examined after 48 and $96 \mathrm{~h}$ [9]. Plates that showed any growth were held until the micro-organisms had been identified. All cultures that showed no growth were incubated for at least 5 days before being discarded. Anaerobic isolates were identified by the API Anaerobic System (Analytab Products, Plainview, NY, USA) or by the Minitek system (BBL). When complete identification was not possible by these methods, other carbohydrate tests (Scott Laboratories, Fiskeville, RI, USA) and gasliquid chromatography (GLC) $[8,9]$ were performed as needed to identify the organisms. The criteria for identification were according to guidelines in published schemes [8-10].

In gram-stained films, Bifidobacterium spp. are generally branched or bifurcate, Eubacterium spp. are pleomorphic and Lactobacillus spp. are straight or curved. Species vary from strictly anaerobic to aerotolerant [8,9]. Bifidobacterium and Lactobacillus spp. are nitrate-positive and indole-negative. Lactobacillus spp. produce lactic acid as the sole end product whereas Bifidobacterium spp. produce acetic and lactic acids (with more of the former than the latter). The genus Eubacterium comprises gram-positive, nonsporing bacilli whose GLC pattern is not characteristic of other genera.

\section{Results}

During the study period, 2033 specimens were examined for anaerobic bacteria; 57 isolates of Bifidobacterium spp., 67 of Eubacterium spp. and 41 of strictly anaerobic Lactobacillus spp. were obtained from various sites that accounted for 1780 specimens. These species were not isolated from other sites, such as bile, joint, bone, sinuses, central nervous system and urinary tract, that accounted for 253 specimens.

\section{Bifidobacterium spp.}

The 57 Bifidobacterium isolates were from 55 patients aged 2 weeks-16 years (mean 5 years and 8 months); 37 were males (Table 1). The isolates comprised 19 $(33 \%)$ B. adolescentis, five $(9 \%)$ B. dentium and 33 $(58 \%)$ other Bifidobacterium spp. Infections were polymicrobial in $53(96 \%)$ patients, but in two $(4 \%)$ a Bifidobacterium spp. was isolated in pure culture; these were an isolate of $B$. adolescentis from chronic otitis media and a Bifidobacterium sp. from cervical adenitis.

Most Bifidobacterium isolates were from chronic otitis media $(23 ; 40 \%)$, abscesses or peritonitis $(7 ; 12 \%$ each), cholesteatoma, aspiration pneumonia and paronychia $(3 ; 5 \%$ each). In the 53 specimens that yielded mixed growth including a Bifidobacterium sp., there were 148 other isolates; $89(60 \%)$ of these were strict anaerobes and $59(40 \%)$ were facultative or aerobic species. The number of isolates in mixed cultures varied from two to five (average 2.8 isolates/specimen; 1.7 anaerobes and 1.1 facultative or aerobic species).

The anaerobic organisms isolated most commonly with Bifidobacterium spp. were Peptostreptococcus spp. (28 isolates), Fusobacterium spp. (17), pigmented Prevotella and Porphyromonas spp. (16) and Bacteroides spp. (6). The most common aerobic and facultative organisms isolated with Bifidobacterium spp. were $\alpha$-haemolytic streptococci (15 isolates), Staphylococcus aureus (9), Escherichia coli (7), Pseudomonas aeruginosa (5) and Streptococcus pyogenes (5). Most Bacteroides and E. coli isolates found in mixed culture with Bifidobacterium spp. were from peritonitis, whereas most Prevotella, Porphyromonas 
Table 1. Significant isolates of Bifidobacterium, Eubacterium and Lactobacillus from clinical specimens in children

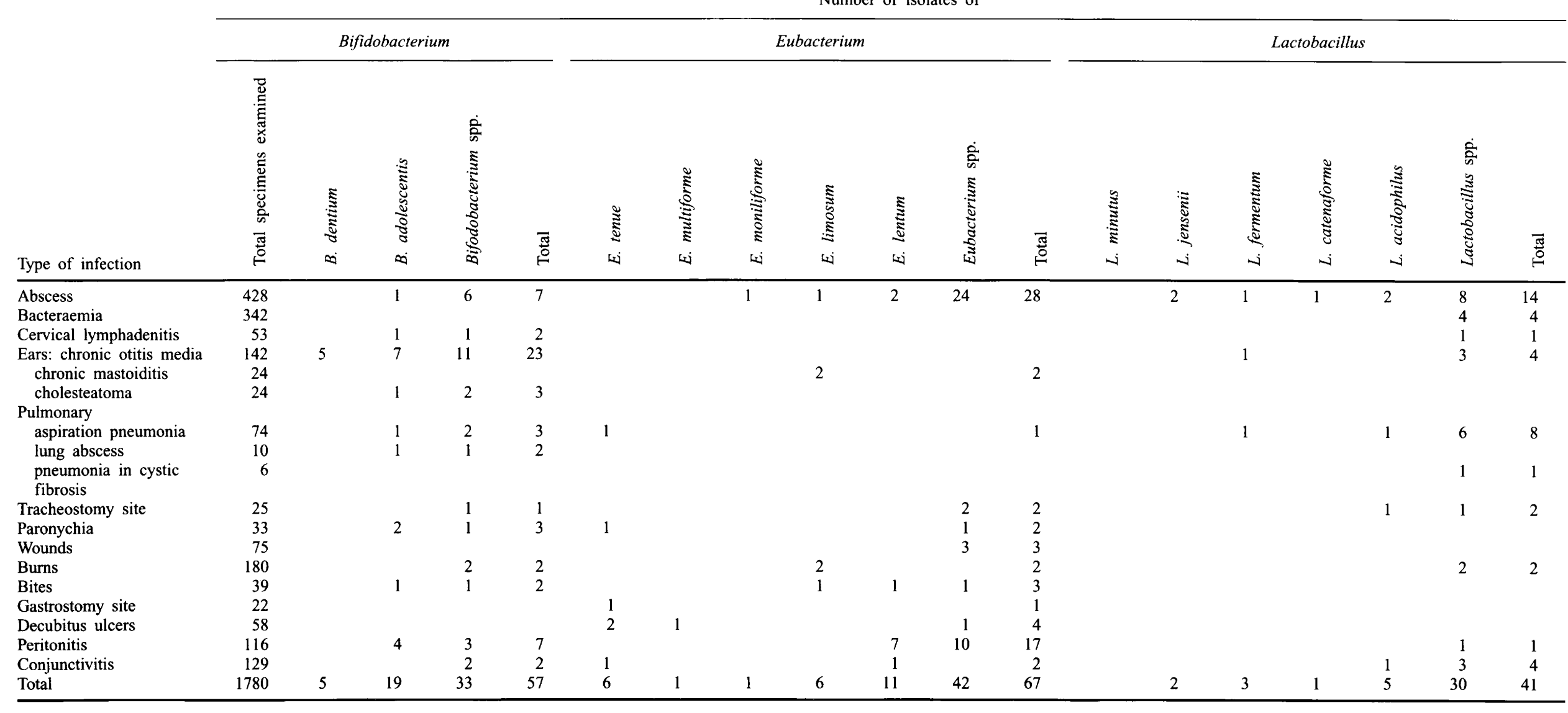


and Fusobacterium spp. were from ear, head and neck and pulmonary sites. Anaerobic cocci were isolated from all sites.

Eleven $(20 \%)$ of the patients had predisposing or underlying medical or surgical conditions. These were previous surgery (5), malignancy (2), steroid therapy (2) and tympanostomy tubes (2). Antimicrobial therapy was administered to all patients, in conjunction with surgical drainage with or without correction of pathology in $21(38 \%)$. The infections resolved in $49(89 \%)$ of the patients. Six $(29 \%)$ of the 21 cases with chronic otitis media did not resolve. The systemic antimicrobial agents given were clindamycin (in 16 instances), a cephalosporin (14), oxacillin (8), co-amoxyclav (8), gentamicin (7), penicillin (4) and chloramphenicol (3). Local therapy with erythromycin ointment was used to treat the two cases of conjunctivitis.

\section{Eubacterium spp.}

The 67 isolates of Eubacterium spp. were from 65 patients aged 7 days-16 years (mean 6 years and 2 months); 39 patients were male (Table 1). The isolates comprised $11(19 \%) E$. lentum, six (11\%) each of $E$. limosum and $E$. tenue, and one ( $2 \%$ ) each of $E$. moniliforme and E. multiforme and $42(74 \%)$ Eubacterium spp. Infections were polymicrobial in 61 (94\%) patients, but in four $(6 \%)$ a Eubacterium sp. was the only isolate. The four isolates found in pure culture were one each of $E$. lentum and Eubacterium sp. in subcutaneous abscesses, and one isolate of a Eubacterium sp. in each of a wound and a burn site.

Most Eubacterium isolates were from abscesses (28; $41 \%$ ), peritonitis $(17 ; 25 \%)$, decubitus ulcers $(4 ; 6 \%)$ and bites $(3 ; 4 \%)$. The 26 abscesses from which eubacteria were isolated were peritonsillar (8), retropharyngeal (4), perirectal (4), dental (3), abdominal (3), subcutaneous (2) and scalp (2). In the 61 specimens that yielded a mixed growth including a Eubacterium sp., there were 152 other isolates; 94 $(62 \%)$ of these were strict anaerobes and $58(38 \%)$ were facultative or aerobic bacteria. The number of isolates in mixed infection cultures varied from two to six (average 2.5 isolates/specimen; 1.5 anaerobes and 1.0 facultative or aerobic). The anaerobic organisms isolated most commonly with eubacteria were Peptostreptococcus spp. (40 isolates), Bacteroides spp. (16), Prevotella and Porphyromonas spp. (15) and Fusobacterium spp. (6). The most common aerobic and facultative organisms isolated with Eubacterium spp. were $S$. aureus (16 isolates), Esch. coli (13), $\alpha$ haemolytic streptococci (12) and Str. pyogenes (5). Most Bacteroides spp. and $E$. coli found in mixed culture with Eubacterium spp. were from peritonitis and abscesses and wounds around the rectal area. Most Prevotella, Porphyromonas and Fusobacterium spp. were isolated from wounds and abscesses around or in the oropharynx.

Twelve $(18 \%)$ of the children had predisposing or underlying medical or surgical conditions. These were previous surgery (4), malignancy (3), steroid therapy (2), diabetes (2) and sickle cell disease (1). Systemic antimicrobial therapy was given to $59(91 \%)$ patients, in conjunction with surgical drainage with or without correction of pathology in $47(72 \%)$ instances. All the infections resolved. The antimicrobial agents given were clindamycin (in 18), a cephalosporin (16), gentamicin (12), oxacillin (9), co-amoxyclav (8), penicillin (6), chloramphenicol (4), erythromycin (3) and ticarcillin-clavulanate (2). Local therapy with an antibiotic or anti-infective ointment was given to two patients with wounds, two with burns and two with conjunctivitis.

\section{Lactobacillus spp.}

The 41 isolates of Lactobacillus spp. were from 40 children aged 8 months-16 years (mean 7 years and 5 months). The isolates were five $(12 \%)$ L. acidophilus, three $(7 \%) L$. fermentum, two $(5 \%) L$. jensenii, one (2\%) L. catenaforme and 30 (75\%) Lactobacillus spp. Infections were polymicrobial in $36(90 \%)$ patients. The four Lactobacillus isolates from blood cultures were in pure culture and were from four patients with intravenous catheter-related bacteraemia.

Most Lactobacillus isolates were from abscesses (14; $34 \%$ ), aspiration pneumonia $(8 ; 20 \%)$, ear infection, bacteraemia and conjunctivitis $(4 ; 10 \%$ each). The 14 abscesses from which Lactobacillus spp. were isolated were dental (4), subcutaneous (3), vulvovaginal (2), abdominal (2), subdiaphragmatic (1), lung (1) and tonsillar (1). In the 36 specimens that yielded mixed growth including a Lactobacillus sp., there were 76 other isolates; $49(64 \%)$ of these were strict anaerobes and $27(36 \%)$ were facultative or aerobic bacteria. The number of isolates in mixed cultures varied from two to five (average 2.1 isolates/specimen, 1.4 anaerobes and 0.7 facultative of aerobic). The anaerobic organisms isolated most often with Lactobacillus spp. were Peptostreptococcus spp. (20 isolates), pigmented Prevotella and Porphyromonas spp. (15) and Fusobacterium spp. (8). The most common aerobic and facultative organisms isolated with Lactobacillus spp. were $S$. aureus (7 isolates), $\alpha$-haemolytic streptococci (6), Haemophilus influenzae (3) and E. coli (2). All Prevotella, Porphyromonas and Fusobacterium spp. were from abscesses and wounds around or in the oropharynx. Most $S$. aureus isolates were from subcutaneous abscesses and wounds, and the $H$. influenzae isolates were from conjunctivitis and otitis.

Eight of the patients had one or two predisposing or underlying medical or surgical conditions. These were intravenous catheter (4), immunodeficiency (3), ma- 
labsorption (2) and malignancy (1). Systemic antimicrobial therapy was given to $35(87 \%)$ patients, in conjunction with drainage with or without correction of pathology in $21(52 \%)$ instances. All the infections resolved. The antimicrobial agents used were clindamycin (12), gentamicin (10), amoxycillin (9), coamoxyclav (7), a cephalosporin (7), ticarcillin-clavulanate (4) and methicillin (3). In addition to systemic antimicrobial therapy, two of the four catheters associated with bacteraemia were removed. The two other patients recovered without the removal of the catheters. Local therapy with ophthalmic ointment containing antimicrobial agents resulted in recovery in the four cases of conjunctivitis.

\section{Discussion}

This review demonstrates the prevalence of Bifidobacterium, Eubacterium and Lactobacillus spp. in various infections in children. The 57 Bifidobacterium isolates were obtained from 55 (3\%) of the 2033 specimens submitted for anaerobic cultures, the 67 isolates of Eubacterium spp. were from 65 (3\%) patients and the 41 Lactobacillus isolates were from $40(2 \%)$ children. These micro-organisms were found most commonly in infections associated with predisposing or underlying conditions such as previous surgery, malignancy, immunodeficiency and the presence of a foreign body.

All three genera of non-sporing gram-positive anaerobic rods were found in abscesses. Bifidobacterium spp. were found mostly in head and neck and pulmonary infections, and in a smaller number of cases of peritonitis. In contrast, Eubacterium spp. were not isolated from chronic otitis media but were more frequent in peritonitis and wounds. Lactobacillus spp. were most commonly found in aspiration pneumonia and bacteraemia associated with intravenous catheters.

Species of the genus Bifidobacterium are part of the commensal flora of the mouth, gastrointestinal tract and female genital tract and constitute a high proportion of the normal intestinal flora in man, especially in breast-fed infants [11]. Although some infections caused by these organisms have been reported [12-15], little is known about their pathogenic potential. Eubacterium spp. are part of the flora of the mouth and the bowel. They have been recognised as pathogens in chronic periodontal disease [16], and in infections associated with intra-uterine devices [3], and have been isolated from patients with bacteraemia associated with malignancy [17] and from female genital tract infection [18]. Lactobacillus spp. are ubiquitous inhabitants of the human oral cavity, the vagina and the gastrointestinal tract [19]. They have been implicated in various serious deep-seated infections, amnionitis [19] and bacteraemia [20].
The isolation of non-sporing anaerobic gram-positive rods from sites of sometimes serious infections in children suggests that they should not be automatically dismissed as contaminants. It appears that they may have clinical significance in high-risk patients with serious underlying illnesses. As they can cause significant infections, especially in high-risk patients, efforts should be made to secure specimens free of contamination by the normal flora of the mucous membranes and the skin, and the clinical significance of each isolate must be carefully evaluated. Eradication of these micro-organisms from deep-seated sites of infection may be difficult. All non-sporing anaerobic gram-positive rods are susceptible to penicillin G, carbenicillin and chloramphenicol [21]. Clindamycin is effective against $94 \%$ of isolates, erythromycin against $88 \%$ and tetracycline against $60 \%$ [22]. Metronidazole is effective against only $50 \%$ of isolates [21-23]. The efficacy of cephalosporins varies between $89 \%$ and $95 \%$.

The assistance of the staff of the microbiology laboratories at the University of California Medical Center, County Medical Center and Serra Memorial Hospital in Los Angeles, Fairview State Hospital in Costa Mesa, and Children's Hospital National Medical Center and South-East Medical Center in Washington, DC, and the Naval Hospital in Bethesda, MD, and the secretarial support of Sarah Blaisdell are gratefully acknowledged.

\section{References}

1. Brook I. Pediatric anaerobic infection: diagnosis and management, 2nd edn. St Louis, Mosby. 1989.

2. Georg LK, Roberstad GW, Brinkman SA, Hicklin MD. A new pathogenic anaerobic Actinomyces species. J Infect Dis 1965; 115: 88-99.

3. Hill GB, Ayers OM, Kohan AP. Characteristics and sites and infection of Eubacterium nodatum, Eubacterium timidum, Eubacterium brachy, and other asaccharolytic eubacteria. $J$ Clin Microbiol 1987; 25: 1540-1545.

4. Bayer AS, Chow AW, Betts, D, Guze LB. Lactobacillemia report of nine cases: important clinical and therapeutic considerations. Am J Med 1978; 64: 808-813.

5. Bourne KA, Beebe JL, Lue YA, Ellner PD. Bacteremia due to Bifidobacterium, Eubacterium or Lactobacillus; twenty-one cases and review of the literature. Yale J Biol Med 1978; 51: 505-512.

6. Finegold SM. Anaerobic bacteria in human disease. New York, Academic Press. 1977.

7. Brook I, Frazier EH. Significant recovery of nonsporulating anaerobic rods from clinical specimens. Clin Infect Dis 1993; 16: $476-480$.

8. Holdeman LV, Cato EP, Moore WEC, eds. Anaerobe laboratory manual, 4th edn. Blacksburg, VA, Anaerobe Laboratory, Virginia Polytechnic Institute and State University. 1977.

9. Summanen P, Baron EJ, Citron DM, Strong CA, Wexler HM, Finegold SM. Wadsworth anaerobic bacteriology manual, 5th edn. Belmont, CA, Star Publishing Company. 1993.

10. Murray PR, Baron EJ, Pfalter MA, Tenover FC, Yolken RH. Manual of clinical microbiology, 6th edn. Washington DC, ASM Press. 1995.

11. Sato J, Mochizuki K, Homma N. Affinity of the Bifidobacterium to intestinal mucosal epithelial cells. Bifidobacteria Microflora 1982; 1: 51-54.

12. Gorbach SL, Thadepalli $\mathrm{H}$. Clindamycin in pure and mixed anaerobic infections. Arch Intern Med 1974; 134: 87-92.

13. O'Connor J, MacCormick DE. Mixed organism peritonitis complicating continuous ambulatory peritoneal dialysis. NZ Med $J$ 1982; 95: 811-812.

14. Thomas AV, Sodeman TH, Bentz RR. Bifidobacterium (Actinomyces) eriksonii infection. Am Rev Respir Dis 1974; 
110: $663-668$.

15. Hata D, Yoshida A, Ohkubo $\mathrm{H}$ et al. Meningitis caused by Bifidobacterium in an infant. Pediatr Infect Dis $J$ 1988; 7 669-671.

16. Vincent JW, Falkler WA, Suzuki JB. Systemic antibody response of clinically characterized patients with antigens of Eubacterium brachy initially and following periodontal therapy. $J$ Periodontol 1986; 57: 625-631.

17. Fainstein V, Elting LS, Bodey GP. Bacteremia caused by nonsporulating anaerobes in cancer patients. A 12-year experience. Medicine (Baltimore) 1989; 68: 151-162.

18. Brook I. Anaerobic bacterial bacteremia: 12-year experience in two military hospitals. J Infect Dis 1989; 160: 1071-1075.

19. Cox SM, Phillips LE, Mercer LJ, Stager CE, Waller S, Faro S Lactobacillemia of amniotic fluid origin. Obstet Gynecol 1986
68: $134-135$.

20. Sherman ME, Albrecht M, DeGirolami PC et al. Lactobacillus: an unusual case of splenic abscess and sepsis in an immunocompromised host. Am J Clin Pathol 1987; 88: 659662.

21. Sutter VL, Finegold SM. Susceptibility of anaerobic bacteria to 23 antimicrobial agents. Antimicrob Agents Chemother 1976; 10: 736-752.

22. Rolfe RD, Finegold SM. Comparative in vitro activity of ceftriaxone against anaerobic bacteria. Antimicrob Agents Chemother 1982; 22: 338-341.

23. Wexler HM, Finegold SM. In vitro activity of cefotetan compared with that of other antimicrobial agents against anaerobic bacteria. Antimicrob Agents Chemother 1988; 32: 601-604. 